\title{
Does the Body Adipose Index (BAI) Work for Elite Athletes? A Comparison of Body Composition Tests Between the Body Adipose Index (BAI) and Bodpod of Female, College Volleyball Players
}

\author{
Marian H. Wooten, PhD \\ Danny Davis, Ed.D. \\ Tommy Thompson, Ed.D. (retired) \\ Whitney Jackson, M.A. candidate \\ The University of North Carolina at Pembroke; \\ Pembroke, North Carolina, USA
}

Doi: 10.19044/esj.2017.c1p3 URL:http://dx.doi.org/10.19044/esj.2017.c1p3

\begin{abstract}
The Body Adipose Index (BAI) is a relatively new equation used to assess an individual's percentage of bodyfat. Two benefits of the BAI are ease and low cost of testing. While research has looked at the BAI for a general population sample, the BAI has not been tested on elite athletes. This limited study compared results of BAI testing with results from the BodPod, a wellestablished and precise body composition analysis device, to find out how well the BAI could measure level of bodyfat in female, collegiate volleyball players. Because the volleyball players had higher fat levels that was assumed when selecting a sample population, findings indicated that the BAI appears to work well on a general female population; however, further research is needed to see if this holds true for a larger sample and whether or not the BAI can measure bodyfat accurately for elite athletes.
\end{abstract}

Keywords: Body Adipose Index (BAI), BodPod, body composition testing, bodyfat

\section{Introduction}

For many years, the Body Mass Index (BMI) has been the common method used to measure and estimate overfatness/obesity, and in fact, the BMI is endorsed by the Centers for Disease Control and Prevention. The BMI is a measure of bodyfat based on a person's weight and height and usually is accompanied by a chart with four ranges within which an individual may fall: Underweight, healthy (normal), overweight, and obese (Liguori \& 
Carrol-Cobb, 2012). Recently some researchers at the University of Southern California (Bergman, Stefanovski, Buchanan, Sumner, Reynolds, Sebring, Xiang, \& Watanabe, 2011) considered such variables as age, sex, height, and weight in determining an accurate way to calculate bodyfat and found that instead of height-weight, height-to-hip circumference provided a more accurate ratio to assess body composition, and they called this new equation the Body Adipose Index (BAI). The equation developed is (hip circumference in $\mathrm{cm} /$ height (in $\mathrm{m})^{1.5}$ )-18.

Bergman, et al (2011) believe that this BAI has promise as a new measurement tool, especially in under-privileged and/or remote settings because it is calculated without using bodyweight and requires minimal measuring protocols (height in meters and hip circumference in centimeters) and a minimal level of training to execute properly. The advantages of BAI include ease, low-tech equipment, and no dependence on bodyweight, instead using only circumference measurements. Bergman, et al (2011) did concede that more research needs to be done before the BAI can be considered a valid tool for assessing bodyfat. Therefore, the purpose of this exploratory study was to test the BAI against the BodPod (recognized as one of the most accurate body composition tools) to determine if there was any correlation in bodyfat measurement, and if so, how much.

\section{I.}

\section{BAI and BodPod}

In their BAI research, Bergman and his colleagues (Bergman, et al, 2011) analyzed data from 1700 adult male and female Mexican-Americans and found that hip circumference and height were strongly linked with bodyfat; a follow-up second study of 220 adult African-Americans found similar results, suggesting that the Body Adipose Index (BAI) may be suitable for all ethnic groups. Although Caucasians were not included, it must be remembered that most of the world's population is non-white. The researchers validated the BAI against dual-energy X-ray absorption [DEXA], and the BAI formula appeared to work for both male and female subjects. Findings revealed that hip circumference $(\mathrm{R}=0.602)$ and height $(\mathrm{R}=-0.524)$ were correlated strongly with percentage of bodyfat (Bergman, et al, 2011). When researchers compared BAI with dual-energy X-ray absorptiometry (DXA) results, the correlation between DXA-derived percentage of adiposity and the BAI in a target population had a reliability of $0.85(\mathrm{R}=0.85)$ with a concordance of C_b-0.9.

The BodPod is accepted universally as a state of the art technological method of accurately measuring bodyfat and is the only device of its kind in the world (www.bodpod). It is practical, commercially available, fast, easy, has no radiation exposure, and is comfortable for subjects. The BodPod uses 
air displacement to measure body volume. Computerized pressure sensors determine the amount of air displaced by the person's body, and body volume is calculated by subtracting the volume of air in a closed chamber with a subject inside it from the volume of air in an empty chamber. BodPod validity is 0.93 when compared to hydrostatic (underwater) weighing (Collins, Millard-Stafford, Sparling, Snow, Rosskopf, Welb, \& Omer, 1999). Furthermore, the BodPod has a reliability of 0.994 with $0.448 \%$ error factor (Collins, et al, 1999) and has been shown to be as reliable as hydrostatic weighing or EDXA in both adults and children (Cornier, Despres, Davis, Grossniklaus, Klein, Lamarche, Lopez-Jimenez, Rao, St-Onge, Towfighi, \& Poirier, 2011).

Beyond its reliability and validity, another benefit of the BodPod is its ease of use when compared to other established measures of bodyfat, like hydrostatic weighing. One major limitation in widespread use of BodPod testing is cost. The equipment used in the exploratory study presented here cost approximately $\$ 50,000$. Another issue is location of the equipment; due to its cost, sensitivity to temperature changes and air movement, and subject comfort (because for testing men must wear form fitting spandex trunks or briefs and women must wear sports bras and spandex shorts or bathing suits), the BodPod needs to be in a relatively quiet environment with controlled access (Fields, Hunter, \& Goran, 2000).

\section{Methods}

Because the goal of this research project was to compare results of BodPod testing to BAI for a healthy population, a female collegiate volleyball team at the NCAA Divison II level was selected since the team was thought accurately to represent adults with low to healthy levels of bodyfat. This particular team was chosen for three reasons: (1) Researchers wanted to look at a single sex, fit population to see how BAI for this sample compared to BAI testing for general population; (2) one member of the research team previously had been a collegiate volleyball coach, hence a personal interest in this particular team; and (3) when apprised of the research that was to occur, the volleyball team and its coaches expressed willingness to serve as test subjects. The team had 14 players; however, due to class schedule conflicts, only 13 members were able to complete both BodPod and BAI testing. The players ranged from freshmen to seniors and all were identified as either Caucasian/white or African-American/black.

All testing occurred in the first half of the 2011 volleyball season. BodPod testing was done two weeks before BAI measurements were taken. The assistant coach took responsibility for scheduling BodPod testing for team members at ten minute intervals. Each subject's BodPod test was conducted in the university exercise performance lab and took approximately 
six minutes per individual. To ensure participant comfort, a female researcher was present for all tests, as was the team's athletic trainer. Height measurements were self-reported for BodPod testing based on the researchers' belief that in a sport such as collegiate volleyball, where height is important and reported frequently, players would be able to report their heights accurately. Each individual wore either form-fitting spandex shorts and a sports bra or a bathing suit, as recommended by previous researchers and instructions accompanying the BodPod (Fields, Hunter, \& Goran, 2000).

BAI measurements were obtained approximately two weeks after BodPod testing prior to the team's regular practice. Because all testing was done during the competitive season, there should not have been any significant changes in the body composition in the study population. Height was measured against a wall, and hip circumference was measured on a horizontal plane at the level of the widest circumference over the buttocks using a cloth tape measure. To ensure participant comfort, a female researcher took hip measurements required for BAI calculation and described the process to each subject as it occurred. For both BodPod and BAI measurements, participants wore the same type shorts, form-fitting spandex shorts, as are required for BodPod testing and typically worn by volleyball players at practice. Therefore, the hip BAI measurement was taken over these shorts.

Using hip and height data obtained during BAI testing, two members of the research team independently calculated BAI. Through a comparison of results, any discrepancies in data were rectified. Then each female's BAI and BodPod measurements were entered into a statistical software package (PASW Statistics 18) for analysis.

\section{Results}

All results of BodPod and BAI testing are expressed as percentages. BodPod results indicated that percent bodyfat for subjects ranged between $17.70 \%$ and $33.80 \%$ with a mean bodyfat of $26.59 \%$ and a standard deviation of $5.20 \%$. Use of the Shapiro-Wilk test revealed that BodPod measurements were normally distributed ( $W=0.953, \mathrm{p}=0.651)$, and this finding was confirmed visually with a normal Q-Q plot.

When using the BAI equation, subjects' bodyfat ranged between $21.66 \%$ and $31.51 \%$ with a mean of $27.34 \%$ and a standard deviation of $3.30 \%$. The Shapiro-Wilk test and normal Q-Q plot were employed to check for normal distribution. Both tests indicated that the data was normally distributed $(W=0.925, \mathrm{p}=0.283)$.

To compare the results from BodPod and BAI tests across subjects, a paired t-test was employed. This test was selected to ascertain whether or not subjects' percent bodyfat differed significantly based on the test used (Ott \& Longnecker, 2001). Results indicated that the mean percent bodyfat from 
BodPod testing was not statistically different from the mean percent bodyfat from BAI testing $(\mathrm{t}=0.726, \mathrm{p}=0.481)$ at the $95 \%$ confidence level.

\section{Conclusion}

\section{Significance of Findings}

Based on this limited study, it appears that the BAI is a fairly reliable measure of bodyfat percentage in a selected group of college female athletes. This confirms findings from Bergman, et al (2011). However, the subjects' range of bodyfat $(21.66 \%-31.51 \%$ with a mean of $27.34 \%$ as calculated using BAI and $17.70 \%-33.80 \%$ with a mean of $26.59 \%$ as calculated by the BodPod) was higher than anticipated for collegiate athletes based on multiple published reports and tables, including those by Kenney, Wilmore, and Costill (2012) and the American Council on Exercise (ACE). Kenney, Wilmore, and Costill (2012) found elite female volleyball players to be between 10-18\% bodyfat. ACE classifies bodyfat ranges for women as follows: $10 \%-13 \%=$ essential fat; $14 \%-20 \%=$ athletes; $21 \%-24 \%$ bodyfat $=$ fitness $; 25-31 \%$ bodyfat = average individuals; and $32 \%$ bodyfat or higher $=$ obese (Muth, 2009). Therefore, this study does not indicate that BAI is a good measure of bodyfat for elite athletes or perhaps even especially fit individuals. However, because the study population carried a higher level of body fat than expected in elite female volleyball players, it appears from this limited research that the BAI could be a practical assessment tool for everyday practitioners to assess percent of bodyfat in such settings as physical education classes, recreational environments (like fitness centers), and catering to the general population.

\section{Limitations}

The main limitation in this study was the limited sample size $(\mathrm{N}=13)$. A larger sample size would have yielded more generalizable results. Had the sample been expanded to include women's volleyball players at other Division II institutions or other female athletes at the same institution as the volleyball team, results may have been different. Another limitation was that BAI and BodPod testing could not be completed for each subject on the same day and in the same attire. However, due to one of the researcher's past experience as a Division II collegiate volleyball coach and the fact that the team was in season when both phases of testing were completed, changes in body composition were assumed to be negligible in the two weeks between BodPod and BAI testing.

\section{Future Research}

While the BAI appears to work well on a general female population, based on the results of this study and those by Bergman, et al (2011), more 
research needs to be done. Bergman et al (2011) did not test the BAI equation on white/Caucasian females; the present research did include white/Caucasian females but did not consider race/ethnicity as a factor in the accuracy of BAI. More BAI research needs to be done, specifically on white/Caucasian females and males. In addition, while this study endeavored to look at elite athletes, it actually investigated females who were not in an elite range. Therefore, research should be done specifically on elite athletes.

\section{References:}

1. Bergman, R. N., Stefanovski, D., Buchanan T. A., Sumner, A. E., Reynolds, J. C., Sebring, N.

2. G., Xiang, A. H., \& Watanabe, R. M. (2011). A better index of body adiposity. Obesity, 19, 1083-1089.

3. BodPod example: Body composition assessment (2014). Retrieved from www.bodpod.com.

4. Collins M. A., Millard-Stafford, M. L., Sparling, P. B., Snow, T. K., Rosskopf, L.B., Welb S. A,. \& Omer, J. (1999). Evaluation of the BodPod for assessing bodyfat in collegiate football players. Medicine and Science in Sports \& Exercise. 31(9): 1350-1356.

5. Cornier, M., Despres, J., Davis, N., Grossniklaus, D.A., Klein, S., Lamarche, B., Lopez-Jimenez, F., Rao, G., St-Onge, M., Towfighi, A., \& Poirier, P. (2011, 26 September). Assessing adiposity: A scientific statement from the American Heart Association. Retrieved from http://www.circ.ahajournals.org/content/0b013e318233.do

6. Fields, D. A., Hunter, G. R., \& Goran, M. I. (2000). Validation of the BOD POD with hydrostatic weighing: Influence of body clothing. International Journal of Obesity, 24, 200-205.

7. Hughes, S. (2010, July 19). Trigylceride and waist measurements predict heart-disease risk. Retrieved from http://www.theheart,org/article/1100179.do

8. Kenney, W. L., Wilmore, J. H., Costill, D. L. (2012). Physiology of sport and exercise $\left(5^{\text {th }}\right.$ ed). Human Kinetics.

9. Liguori, G. \& Carrol-Cobb, S. (2012). FitWell. McGraw-Hill.

10. McCrory, M.A., Gomez, T.D., Bernauer, E.M., \& Mole, P.A. (1995). Evaluation of a new air displacement plethysmograph for measuring human body composition. Medicine \& Science in Sports \& Exercise. 27 (12), 1686-1691.

11. Muth, N. D. (2009). What are the guidelines for percentage of body fat loss? Fit Life. Retrieved from http://www.acefitness.org/acefit/healthy-living-article/60/112/whatare-the-guidelines-for-percentage-of/ 
12. Nainggolan, L. (2011, February 23). BMI-death link is same for Asians as Europeans. Retrieved from http://www.theheart.org/article/1188959.do

13. Nainggolan, L. (2009, December 7). Body-mass index and waist circumference equally predictive for $\mathrm{CV}$ events, mortality. Retrieved from http://www.theheart.org/article/1032175.do

14. Ott, R. L., \& Longnecker, M. (2001). An introduction to statistical methods and data analysis ( $5^{\text {th }}$ ed.). Duxbury.

15. Wood, S. (2007, December 11). More support for waist-to-hip ratio over BMI to predict CHD. Retrieved from http://www.theheart.org/article/832669.do

16. Wood, S. (2010, May 10). Adding height to waist/hip ratio increases mortality prediction, but who will use it? Retrieved from http://www.theheart.org/article/1076749.do 